\title{
CLASSIFICATION OF THE FINITE NONLINEAR PRIMITIVE LIE ALGEBRAS
}

\author{
BY \\ TAKUSHIRO OCHIAI
}

0 . Introduction. Let $L$ be a finite dimensional Lie algebra over the field of real or complex numbers and $L_{0}$ be a subalgebra of $L$. The pair $\left(L, L_{0}\right)$ is called a transitive Lie algebra if the following condition is satisfied;

(p-1) $L_{0}$ does not contain any nonzero ideal of $L$. A transitive Lie algebra $\left(L, L_{0}\right)$ is called nonlinear primitive $\left({ }^{1}\right)$ if the following conditions are satisfied;

(p-2) $L_{0}$ is a maximal subalgebra of $L$;

(p-3) the subset $L_{1}$ (called the derived algebra of $L_{0}$ ), defined by

$$
L_{1}=\left\{t \in L_{0} \mid[t, L] \subseteq L_{0}\right\}
$$

is not $\{0\}$. A transitive Lie algebra $\left(L, L_{0}\right)$ is called nonlinear irreducible $\left.{ }^{1}\right)$ if it satisfies (p-3) and the following (p-4);

(p-4) if there is a subspace $M$ of $L$ such that $L \supseteq M \supseteq L_{0}$ and $\left[L_{0}, M\right] \subseteq M$, then either $M=L$ or $M=L_{0}$.

It is immediate that any irreducible Lie algebra is primitive. Two transitive Lie algebras $\left(L, L_{0}\right)$ and $\left(L^{\prime}, L_{0}^{\prime}\right)$ are isomorphic if there exists a Lie algebra isomorphism $\phi$ of $L$ onto $L^{\prime}$ such that $\phi\left(L_{0}\right)=L_{0}^{\prime}$.

In this paper we will give the classification of the nonlinear primitive Lie algebras up to isomorphism (§3). Recently S. Kobayashi and T. Nagano completely classified the nonlinear irreducible Lie algebras up to isomorphism [4]. Our results naturally include their results. We will give some criterion for a nonlinear primitive Lie algebra to be irreducible (Propositions 7, 8, and $8^{\prime}$ ) after introducing a kind of the structure theorem of the nonlinear primitive Lie algebras ( $\$ 4)$. Lemma 6 in $[4$, p. 881$]$ is an immediate consequence of our structure theorem. (Lemma 6 in [4] is of some importance for the theory of the analytic Lie pseudogroups [12].)

E. Cartan says that he has classified the nonlinear primitive Lie algebras [2], but his result is completely different from ours. His method seems to be wrong. That is to say, his Theorem II in [2, p. 101], which he also used to classify the infinite primitive Lie algebras [5], seems to be wrong, as was pointed out by I.M. Singer and S. Sternberg [12]. We will give a counterexample to his proof of

Received by the editors November 1, 1965.

(1) When $\left(L, L_{0}\right)$ satisfies (p-2) (resp. (p-4)), it is called primitive (resp. irreducible). 
Theorem II ( $\$ 5)$. It will be necessary to remark that this paper has nothing to do with the classification of the infinite primitive Lie algebras [5]. But we hope that this paper gives some data for that big problem [3], [12]. For that purpose we will give a few remarks in the last section ( $\$ 5)$.

In this paper all the vector spaces will be finite dimensional unless otherwise stated. The author wishes to thank Professor T. Nagano, for his valuable suggestions and criticisms, Mr. M. Takeuchi and Mr. S. Kaneyuki. He owes this paper to discussions with them. He also wishes to thank Professor S. Kobayashi.

1. Let $\left(L, L_{0}\right)$ be a nonlinear primitive Lie algebra. It is easy to see:

LEMMA 1. (i) $L_{0} \neq L_{1}$;

(ii) $\left[L_{0}, L_{1}\right] \subseteq L_{1}$;

(iii) $\left[L, L_{1}\right] \subseteq L_{0}$.

Proof. They are immediate from the definition ( $\$ 0)$.

Next two lemmas are proved in [4, Lemmas 1,2] under the assumption of $\left(L, L_{0}\right)$ being nonlinear irreducible. But simple observation will show that the irreducibility is unnecessary. For completeness we will here give them as lemmas.

LEMma 2. Let $\left(L, L_{0}\right)$ be a nonlinear primitive Lie algebra. Then we have:

(i) If $J$ is a nonzero ideal of $L$, then $L=J+L_{0}$ (not necessarily direct sum);

(ii) if $J_{1}$ and $J_{2}$ are ideals of $L$ such that $J_{1} \cap L_{0} \neq\{0\}$ and $J_{2} \neq\{0\}$, then $\left[J_{1}, J_{2}\right] \neq\{0\}$ (here, $J_{1}$ and $J_{2}$ may be identical);

(iii) if $J$ is a nonzero ideal of $L$, then $J \cap L_{0} \neq\{0\}$;

(iv) L has no abelian ideals other than $\{0\}$.

Proof. (i) Set $S=J+L_{0}$. Since $S$ is a subalgebra of $L$, either $S=L$ or $S=L_{0}$ by (p-2). If $S=L_{0}$, i.e. $J \subseteq L_{0}$, this contradicts (p-1). (ii) Applying the result (i) to $J_{2}$, we have $L=J_{2}+L_{0}$. If $\left[J_{1}, J_{2}\right]=0$, then $J_{1} \cap L_{0}(\neq\{0\})$ is a nonzero ideal of $L$, contradicting (p-1). (iii) Applying the result (i) to $J$, we have $L=J+L_{0}$. If $\left[J, L_{1}\right]=0$, then we have $\left[L, L_{1}\right] \subseteq L_{1}$ by Lemma 1 , i.e., $L_{1}$ is an ideal of $L$, contradicting $(\mathrm{p}-1)$. Therefore $\left[J, L_{1}\right] \neq 0$. On the other hand, $\left[J, L_{1}\right]$ is contained in both $J$ (as $J$ is an ideal) and $L_{0}$ (by Lemma 1). (iv) This is immediate from (ii) and (iii). Q.E.D.

Lemma 3. Let $\left(L, L_{0}\right)$ be a nonlinear primitive Lie algebra. Then

(i) L is a noncompact simple Lie algebra;

(ii) $L_{1}$ is an abelian ideal of $L_{0}$.

Proof. (i) Assume that $L$ is not semisimple. Then there must be a nonzero abelian ideal in $L$ in contradiction to Lemma 2 . If $L$ is not simple, then there exist ideals $J_{1}$ and $J_{2}$ such that $\left[J_{1}, J_{2}\right]=0$ in contradiction to Lemma 2.

Before proving the noncompactness of $L$, we will prove (ii). We define the subspaces $L_{p}(p \geqq 1)$ of $L$ inductively by $L_{p}=\left\{t \in L_{p-1} \mid[t, L] \subseteq L_{p-1}\right\}$. It is easy 
to see that $\left[L, L_{p}\right] \subseteq L_{p-1},\left[L_{p}, L_{q}\right] \subseteq L_{p+q}$. By $(\mathrm{p}-1)$, we have $L_{p-1} \neq L_{p}(p \geqq 2)$ if $L_{p-1} \neq 0$. Therefore, $L$ being of finite dimension, we have $L_{p_{0}} \neq 0, L_{p_{0}+1}=0$ for some positive integer $p_{0}$. Suppose $p_{0} \geqq 2$. Since we then have

$$
\left[L,\left[L_{p_{0}}, L_{q}\right]\right] \subseteq L_{q+1},
$$

it follows that $\operatorname{ad} x \cdot \operatorname{ad} y$ is nilpotent and thus $\operatorname{tr} \operatorname{ad} x \operatorname{ad} y \equiv 0$ for any $x \in L$, $y \in L_{p_{0}}$. $L$ being simple, the Killing form of $L$ is nondegenerate. Hence $p_{0} \leqq 1$, i.e., $L_{2}=0$. We know $\left[L_{1}, L_{1}\right] \subseteq L_{2}$. By the proof of (ii), we see that the Killing form is identically zero on $L_{1}$. Therefore $L$ is not compact, because the Killing form of any compact semisimple Lie algebra is negative definite.

COROLlaRY 4. Let the notations be as before. Then the radical of $L_{0}$ is noncompact.

Proof. Since $L_{1}$ is an abelian ideal in $L_{0}, L_{1}$ is contained in $R$. If $R$ is compact, then the Killing form of $L$ must be negative definite on $R$. But we know that the Killing form of $L$ is identically zero on $L_{1}$. This is a contradiction.

Remark. The discussion above shows that any finite nonlinear primitive Lie algebra is of order 2 [4], [5]. For the geometric interpretation of this, see also [5].

2. Lemma 3 makes the work of the classification very much accessible. To describe the classification, we need the notion of the parabolic subalgebras of semisimple Lie algebras, and the knowledge of the maximal subalgebras of semisimple Lie algebras. In this section we will briefly recall them without any proof. For the details, see [1], [7], [8], [9], [10] and [11].

Let $L$ be a complex semisimple Lie algebra. A subalgebra of $L$ is called parabolic if it contains a maximal solvable subalgebra of $L$. Let $H$ be a Cartan subalgebra of $L$ and $\Delta$ be the set of all nonzero roots of $L$ with respect to $H$. We fix once and for all a root vector $X_{\alpha}$ of a root $\alpha \in \Delta$. Let $\Pi=\left\{\alpha_{1}, \cdots, \alpha_{l}\right\}$ (resp. $\Delta^{+}$) be the fundamental roots system (resp. all the positive roots) with respect to some fixed order. For any subset $E$ of $\Pi$, we define the subset $\Delta(E)$ of $\Delta$ by $\Delta(E)=\Delta \cap Z\{\Pi$ $-E\}$. Here $Z\{\Pi-E\}$ means all of the linear combinations of the roots $\Pi-E$ with integral coefficients. The subalgebra $B=H+\Sigma_{\alpha \in \Delta^{+}} C X_{\alpha}$ is a maximal solvable subalgebra of $L$. It is easy to see that

$$
L(E)=H+\sum_{\alpha \in \Delta^{+}} C X+\sum_{\beta \in \Delta(E)} C X_{-\beta}
$$

is a parabolic subalgebra. Conversely any parabolic subalgebra of $L$ is isomorphic with one of such subalgebras (by an inner automorphism of $L$ ). It is well known that two parabolic subalgebras $L(E)$ and $L\left(E^{\prime}\right)\left(E, E^{\prime} \subseteq \Pi\right)$ are isomorphic if and only if there exists an automorphism $\phi$ of the Dynkin diagram $\Pi$ such that $\phi(E)=E^{\prime}$. Let $\tilde{\mathscr{D}}$ be the set of the pairs $\{\Pi, E\}$ such that $\Pi$ is the Dynkin diagram of 
semisimple Lie algebras and $E$ are subsets of $\Pi$. We classify $\tilde{\mathscr{D}}$ up to an automorphism of the diagram cited above (denoted by $\tilde{\mathscr{D}} / \sim$ ). By the fact mentioned above, there is a one-to-one correspondence between $\tilde{\mathscr{D}} / \sim$ and the isomorphic classes of the pairs $(L, P)$ such that $L$ is a complex semisimple Lie algebra and $P$ is a parabolic subalgebra of $L$ (here, an isomorphism means that cited in $\S 0$ ). It is clear that $L(E)(E \subseteq \Pi)$ is maximal in $L$ if and only if $E$ consists of one simple root.

REMARK. If $L(E)$ and $L\left(E^{\prime}\right)\left(E, E^{\prime} \subseteq \Pi\right)$ are isomorphic, they must be so by an outer automorphism of $L$.

Now we will mention the real case (i.e. the real parabolic subalgebras of the real semisimple Lie algebras). For the description, we need the notion of the Satake diagrams of the real semisimple Lie algebras, we assume the reader to be familiar with it. For the details, see [1], [7].

Let $G$ be a real semisimple Lie algebra. A subalgebra of $G$ is called parabolic if its complexification is parabolic in the complexification $L$ of $G$, (which is also semisimple). Let $\tilde{\mathscr{S}}$ be the set of the pairs $\{\pi, E\}$ such that $\pi$ are the Satake diagram of some real semisimple Lie algebras and $E$ are the subsets of its restricted fundamental root systems [7], [9]. Two elements $\{\pi, E\}$ and $\left\{\pi^{\prime}, E^{\prime}\right\}$ are called isomorphic if there exists an automorphism (of the Satake diagram) $\phi$ from $\pi$ to $\pi^{\prime}$ such that $\phi(E)=E^{\prime}$. Let $\tilde{\mathscr{S}} / \sim$ be the equivalence classes of $\tilde{\mathscr{S}}$ by the isomorphism. It is well known that there is a one-to-one correspondence between $\tilde{\mathscr{S}} / \sim$ and the isomorphic class of the pair $(G, P)$ such that $G$ is a real semisimple Lie algebra and $P$ is a parabolic subalgebra of $G$ (here, the isomorphism is that cited in $\S 0$ ) [7], [9].

Next we will recall the theorems of Morozov and Mostow on the maximal subalgebras of the Lie algebras.

THEOREM A (MoROzov). Let $S$ be a maximal nonsemisimple subalgebra of $a$ complex semisimple Lie algebra L. Then $S$ is a maximal parabolic subalgebra of $L[11]$.

THEOREM B (Mostow). Let $G$ be a real semisimple Lie algebra. Any maximal subalgebra of $G$, the radical of which is noncompact, is a maximal parabolic subalgebra of $G[10]$.

3. In this section we will give the classification of the nonlinear primitive Lie algebras over the real or complex field.

LEMMA 5. Let $(L, P)$ be a pair of a complex semisimple Lie algebra $L$ and a parabolic subalgebra $P$ of L. Then the derived subalgebra $P_{1}=\{t \in P \mid[t, L] \subset P\}$ is not 0.

Proof. We may assume $P=L(E)$ for some subset of the fundamental root system $\Pi$ of $L$ (see $\S 2)$. We denote by $\theta$ the highest root with respect to $\Pi$. Since $P$ contains all the root vectors associated with positive roots and the Cartan sub- 
algebra, $P$ contains $X_{\theta}$, it is easy to see that $\left[L, X_{\theta}\right] \subseteq P$. Thus $P_{1}$ is not 0 since it contains $X_{\boldsymbol{\theta}}$.

Combining Lemma 3, Theorem A, Theorem B and Lemma 5, we obtain

TheORem C. A complex transitive Lie algebra $\left(L, L_{0}\right)$ is nonlinear primitive if and only if

(i) $L$ is a complex simple Lie algebra, and

(ii) $L_{0}$ is a maximal parabolic subalgebra of $L$.

THEOREM D. A real transitive Lie algebra $\left(G, G_{0}\right)$ is nonlinear primitive if and only if

(i) $G$ is a noncompact real simple Lie algebra, and

(ii) $G_{0}$ is a maximal parabolic subalgebra of $G$.

Proof of Theorem C. By (ii) of Lemma 3, $L_{0}$ is not semisimple. Therefore the "only if" part is immediate from (i) of Lemma 3 and Theorem A. By the definition, the "if" part only needs the verification of the derived subalgebra $L_{1}$ being not 0 , which is immediate from Lemma 5.

Proof of Theorem D. The "only if" part is the direct consequence of Corollary 4 and Theorem $B$.

Let $L$ (resp. $L_{0}$ ) be the complexification of $G$ (resp. $G_{0}$ ). By the definition, $L_{0}$ is parabolic in $L$. Thus $\left(L, L_{0}\right)$ satisfies the assumption of Lemma 5 , which says the derived subalgebra $L_{1}$ is not 0 . Now $L_{1}$ is invariant under the involutive automorphism of $L$ associated with $G$ in $L$ since $L_{0}$ is clearly invariant by it. Hence $L$ is the complexification of the derived subalgebra of $G_{1}$. Thus we have $G_{1} \neq 0$, which proves the "if" part. Q.E.D.

Let $\mathscr{D}$ be the subset of $\tilde{\mathscr{D}} / \sim$ which consists of all the elements which have the representatives $\{\Pi, E\}$ such that $\Pi$ are simple $\left({ }^{2}\right)$ and $E$ are single simple roots of $\Pi$. Similarly let $\mathscr{S}$ be the subset of $\tilde{\mathscr{S}} / \sim$ which consists of all the elements which have the representatives $\{\pi, E\}$ such that $\pi$ are simple $\left(^{3}\right)$ and $E$ are single simple roots of the restricted fundamental root systems.

Combining Theorem C, Theorem D and the facts mentioned in $\$ 2$, we obtain the following classification theorems.

THEOREM C'. There is a one-to-one correspondence between $\mathscr{D}$ and the isomorphic classes of the complex nonlinear primitive Lie algebras.

THEOREM $\mathrm{D}^{\prime}$. There is a one-to-one correspondence between $\mathscr{S}$ and the isomorphic classes of the real nonlinear primitive Lie algebras.

4. In this section we will give a kind of structure theorem of the complex nonlinear primitive Lie algebras. Using that theorem, we shall give a criterion for an

(2) If $\Pi$ corresponds to some complex simple Lie algebra, we say $\Pi$ is simple.

(3) If $\pi$ corresponds to some real simple Lie algebra, we say $\pi$ is simple. 
element of $\mathscr{D}$ (resp. $\mathscr{S}$ ) to correspond to the isomorphic class of the complex (resp. real) nonlinear irreducible Lie algebras. This gives the result of $\$ 6$ in [4].

Let $(\Pi, \alpha)$ be any element of $\mathscr{D}$. Choose a complex simple Lie algebra $L$ such that its Dynkin diagram is $\Pi$, and fix it once and for all. Let $H$ be a Cartan subalgebra of $L$ and $\Delta$ be the root system of $L$ with respect to $H$. We may assume that $\Pi$ is the fundamental root system of $\Delta$ with respect to some order. Then the nonlinear primitive Lie algebra $(L, L(\alpha))$ (see Theorem $C$ ) is the representative of the isomorphic class corresponding to $(\Pi, \alpha)$. For each root $\beta \in \Delta$, we can express $\beta$ as the linear combination of the roots of $\Pi$; say

$$
\beta=\sum_{\alpha_{j} \in \Pi} m_{j} \alpha_{j}, \quad m_{j} \text { : integer. }
$$

Let $\rho(\beta)$ be the coefficient of $\alpha$ in the expression (\#). We define the mapping $\rho_{\alpha}$ from $\Delta$ into the integers by applying $\beta$ into $\rho(\beta)$. By the well-known fact, there exists the strictly positive integer $m_{\alpha}$ such that $-m_{\alpha} \leqq \rho_{\alpha}(\beta) \leqq m_{\alpha}$ for any $\beta \in \Delta$. Let $\Delta^{+}$(resp. $\Delta^{-}$) be the set of all positive (resp. negative) roots of $\Delta$ with respect to the order stated before, and $\theta$ be the highest root of $\Delta$. Then $\rho_{\alpha}(\theta)=m_{\alpha}$. We fix, once for all, the root vectors. We define the subspaces $L_{(-1)}, M_{(-1)}, S, \tilde{L}_{1}$ and $\tilde{M}$ as follows.

$L_{(-1)} \quad$ is spanned by those root vectors $X_{\beta}$ such that $\rho_{\alpha}(\beta)<0$;

$M_{(-1)}$ is 0 if $m=1$ and otherwise spanned by those $X_{\beta}$ such that $-\left(m_{\alpha}-1\right)$ $\leqq \rho_{\alpha}(\beta)<0$ if $m_{\alpha} \geqq 2$;

$S \quad$ is spanned by $H$ and by those $X_{\beta}$ such that $\rho_{\alpha}(\beta)=0$;

$\tilde{L}_{1} \quad$ is spanned by those $X_{\beta}$ such that $\rho_{\alpha}(\beta)=m_{\alpha}$, and

$\tilde{M} \quad$ is 0 if $m_{\alpha}=1$ and otherwise spanned by those $X_{\beta}$ such that $0<\rho_{\alpha}(\beta)$ $\leqq m_{\alpha}-1$.

By the construction, $L_{0}=S+\tilde{M}+\tilde{L}_{1}, L=L_{(-1)}+L_{0}$. Now we will prove:

Proposition 6. $L_{1}$ is identical to $\tilde{L}_{1}$.

Proof. We shall prove $L_{1} \supseteq \tilde{L}_{1}$. Let $\beta, \gamma$ be the roots such that $\rho_{\alpha}(\beta)=m_{\alpha}$, $\rho_{\alpha}(\gamma)<0$. If $\beta+\gamma$ is a root, then $\rho_{\alpha}(\beta+\gamma) \geqq 0$. This means $\left[L_{(-1)}, \tilde{L}_{1}\right] \subseteq L_{0}$. Hence $\left[L, \tilde{L}_{1}\right] \subseteq L_{0}$, which proves our assertion. We will prove the converse. Since $L_{0} \supseteq H$, we have $\left[H, L_{1}\right] \subseteq L_{1}$ by Lemma 1 . Hence $L_{1}$ is spanned by the root vectors. $\left(L_{1}\right.$ does not meet $H$ since $\left[L, L_{1}\right] \subseteq L_{0}$.) Let $X_{\gamma}$ be any root vector in $L_{1}$. We will prove $\rho_{\alpha}(\gamma)=m_{\alpha}$. Suppose $\rho_{\alpha}(\gamma) \leqq m_{\alpha}-1$. Firstly we will show that there exists $X_{\gamma^{\prime}} \in L_{1}$ such that $\rho_{\alpha}\left(\gamma^{\prime}\right)=\rho_{\alpha}(\gamma)$ and $\gamma^{\prime}+\alpha$ is a root. In fact, if $\gamma+\alpha$ is not a root, there exist positive roots $\beta_{1}, \beta_{2}, \cdots, \beta_{n}$ different from $\alpha$, such that $\gamma^{\prime}=\left(\cdots\left(\left(\gamma+\beta_{1}\right)+\beta_{2}\right) \cdots \beta_{n}\right)$ is a root and $\gamma^{\prime}+\alpha$ is also a root. Since any $X_{\beta}$ $\left(\beta \in \Delta^{+}\right)$is contained in $L_{0}$ and we have $\left[L_{0}, L_{1}\right] \subset L_{1}$, we see $X_{\gamma^{\prime}} \in L_{1}$. Hence $\left.\left.\left[X_{\gamma^{\prime}}, X_{-\left(\gamma^{\prime}+\alpha\right)}\right] \in\right\} X_{-\alpha}\right\} \nsubseteq L_{0}$. This is a contradiction. Hence $\rho_{\alpha}(\gamma)=m_{\alpha}$.

Proposition 7. Let the notations be as before. $(L, L(\alpha))$ is irreducible if and only if $m_{\alpha} \mid=1$. 
Proof. The "only-if" part: If $m_{\alpha} \geqq 2$, then it is easy to see that $M_{(-1)}+L_{0}$ is a subspace such that $\left[L_{0}, M_{(-1)}+L_{0}\right] \subseteq M_{(-1)}+L_{0}$. This is a contradiction. The "if" part: If $m_{\alpha}=1$, any root vector $X_{\beta}$ such that $\rho_{\alpha}(\beta)=-1$, can be obtained from $X_{-\theta}$ in the sense that $\left\{X_{\beta}\right\} \ni\left[X_{\beta_{m}}\left[X_{\beta_{m-1}}\left[\cdots\left[X_{\beta_{1}}, X_{-\theta}\right] \cdots\right]\right]\right]$ such that $\beta_{j}(1 \leqq j \leqq m) \in \Delta^{+}$and $\rho_{\alpha}\left(\beta_{j}\right)(1 \leqq j \leqq m)=0$ i.e. $X_{\beta_{j}} \in L_{0}$, and vice versa. Since any $M$ such that $\left[L_{0}, M\right] \subseteq M$ is spanned by the root vectors, our assertion has been proved.

Let $(\pi, \mu)$ be an element of $\mathscr{S}$. We choose one representative $(G, G(\mu))$ of the isomorphic class, corresponding to $(\pi, \mu)$ and fix it once for all. We denote by $L$ (resp. $L(\mu)$ ) the complexification of $G$ (resp. $G(\mu)$ ). We must consider two cases;

Case (i). $\quad L$ is simple.

Case (ii). $L$ is not simple.

Case (i). We will prove that $(G, G(\mu))$ is irreducible if and only if $(L, L(\mu))$ is an irreducible Lie algebra. The "if" part is trivial. Suppose that $(G, G(\mu))$ is irreducible and that $(L, L(\mu))$ is reducible $\left({ }^{4}\right)$ (may be not necessarily primitive). Let $M$ be a subspace of $L$ such that $L \supset M \supset L(\mu),[L(\mu), M] \subseteq M$. Let $\sigma$ be the involutive automorphism of $L$ associated with $G$ in $L$. We have only to show that $\sigma(M)+M \neq L$. Since $M$ is spanned by the root vectors and $L_{0}$ contains all the root vectors associated with the positive roots, we have $X_{-\theta} \notin M$. There are two cases:

(a) $L(\mu)=L(\alpha)$ for some simple root;

(b) $L(\mu)=L(\alpha ; \hat{\alpha})$ i.e. $\alpha$ and $\alpha$ are joined by an arrow in the Satake diagram.

Case (a). We put $m_{\alpha}=\rho_{\alpha}(\theta)$. Then $\rho_{\alpha}(\sigma(\theta))=m_{\alpha}[1]$. If $\sigma\left(X_{-\theta}\right) \in M$, then by applying the element of $L(\mu)$, we get $X_{-\theta} \in M$ (remark $\rho_{\alpha}(\sigma(\theta))=m_{\alpha}$ ). This contradicts $X_{-\theta} \notin M$.

Case (b). It is known $\rho_{\alpha}(\theta)=\rho_{\hat{Q}}(\theta)$ [1], and $\sigma(\alpha)=\hat{\alpha}+\gamma$ such that $\rho_{\alpha}(\gamma)=0$ and $\sigma(\gamma)=-\gamma$. Therefore $\rho_{\alpha}(\sigma(\theta))=m_{\alpha}, \rho_{\alpha}(\sigma(\theta))=m_{2}$. If $\sigma\left(X_{-\theta}\right) \in M$, then by applying the elements of $L(\mu)$, we get $X_{-\theta} \in M$. This is a contradiction.

Case (ii). We can consider that $G$ is a complex simple Lie algebra and $G(\mu)$ is its maximal parabolic subalgebra. If $(G, G(\mu))$ is irreducible, then clearly $(G, G(\mu))$ is irreducible as the complex primitive Lie algebra. Conversely suppose $(G, G(\mu))$ is irreducible as the complex primitive Lie algebra. Let $M$ be a real subspace of $G$ such that $[G(\mu), M] \subseteq M$. Then $(-1)^{1 / 2} M \supseteq(-1)^{1 / 2}[G(\mu), M]$ $=\left[(-1)^{1 / 2} G(\mu), M\right]=[G(\mu), M]$. If $(-1)^{1 / 2} M \cap M=G(\mu)$, then $[G(\mu), M]$ $\subseteq M \cap(-1)^{1 / 2} M=G(\mu)$. Hence $\left[G(\mu),(-1)^{1 / 2} M\right] \subseteq(-1)^{1 / 2} G(\mu)=G(\mu)$. Therefore we obtain complex subspace $M+(-1)^{1 / 2} M(\supset G(\mu))$ such that $\left[G(\mu), M+(-1)^{1 / 2} M\right] \subseteq G(\mu)$, by the hypothesis of our assertion $M+(-1)^{1 / 2} M$ $=L$. Hence $[G(\mu), L]=\left[G(\mu), M+(-1)^{1 / 2} M\right] \subseteq G(\mu)$ contradicting (p-1).

(4) There exists a subspace $M$ of $L$ such that $L \supset M \supset L(\mu)$ and $[L(\mu), M] \subseteq M$. 
Hence $(-1)^{1 / 2} M \cap M \nsubseteq G(\mu)$ in contradiction to the irreducibility of $(G, G(\mu))$ as the complex primitive Lie algebra. Thus we have proved:

Proposition 8. Let $(\pi, \mu)$ be any element of $\mathscr{S}$ such that $\pi$ is irreducible (i.e. $\pi$ is the real form of some complex simple Lie algebra). Then $(\pi, \mu)$ is irreducible if and only if $\mu$ is a "white vertex" $\alpha$ [7], which is not joined by an arrow with any "white vertex," and $m_{\alpha}=1$.

Proposition $8^{\prime}$. Let $(\pi, \mu)$ be any element of $\mathscr{S}$ such that $\pi$ is not irreducible (i.e. $\pi$ is the restriction of the base field of some complex simple Lie algebra). Then $(\pi, \mu)$ is irreducible if and only if $\mu$ is the "white vertex" $\alpha$ such that $m_{\alpha}=1$.

From the discussion above we easily see;

COROllary 9. Let $\left(L, L_{0}\right)$ be a complex nonlinear primitive Lie algebra which is reducible. Then the subspace $M$ such that $\left[L_{0}, M\right] \subseteq M$ is characterized by an integer $k$ such that $0 \leqq k \leqq m_{\alpha}$, i.e., $M$ is spanned by the root vectors $X_{\gamma}$ such that $-k \leqq \rho(\gamma) \leqq 0$.

5. In this section we shall give a simple example and show that E. Cartan's proof of the Theorem II (see $\S 0$ ) is wrong, as was pointed out by I. M. Singer and S. Sternberg [12, p. 57]. Also we will give several results.

Let $L$ be the complex exceptional simple Lie algebra of the type $G_{2}$. We denote its two simple roots by $\alpha, \beta$. Then the positive roots $\Delta$ of $L$ consists of $\alpha, \beta$, $\alpha+\beta, 2 \alpha+\beta, 3 \alpha+\beta$ and $3 \alpha+2 \beta$. Consider $(L, L(\beta))$ for example. Then $(\S 4)$

$$
\begin{aligned}
L_{(-1)} & =\left\{X_{-\beta}, X_{-\beta-\alpha}, X_{-\beta-2 \alpha}, X_{-\beta-3 \alpha}, X_{-2 \beta-3 \alpha}\right\} ; \\
M_{(-1)} & =\left\{X_{-\beta}, X_{-\beta-\alpha}, X_{-\beta-2 \alpha}, X_{-\beta-3 \alpha}\right\} ; \\
L_{0} & =\left\{H, X_{-\alpha}, X_{\alpha}, X_{\beta}, X_{\alpha+\beta}, X_{2 \alpha+\beta}, X_{3 \alpha+\beta}, X_{3 \alpha+2 \beta}\right\} ;
\end{aligned}
$$

and

$$
L_{1}=\left\{X_{2 \beta+3 \alpha}\right\} \text {. }
$$

It is easy to see $\left[M_{(-1)},\left[L_{1}, M_{(-1)}\right]\right] \subseteq L_{0}$. Hence this gives us a counterexample to the following lemma of Cartan for the proof of Theorem II.

LEMMA (E. CARTAN). Let $\left(L, L_{0}\right)$ be a nonlinear primitive Lie algebra and $M$ be the subspace of $L$ such that $L \supset M \supset L_{0},\left[L_{0}, M\right] \subseteq M$. Then we have $\left[M,\left[L_{1}, M\right]\right] \neq L_{0}$.

On the other hand we can easily prove:

Proposition 10. Let $\left(L, L_{0}\right)$ be a nonlinear primitive transitive Lie algebra and $M$ be the subspace of $L$ such that $L \supset M \supset L_{0}, \quad\left[L_{0}, M\right] \subseteq M$. Then $\left[L,\left[L_{1}, L\right]\right] \neq M$. 
Proof. Let $\theta$ be the highest root. Then $L$ contains $X_{\theta}, M$ does not contain $X_{-\theta}$ (see $\S 4$ ). Since $\left[X_{-\theta}, X_{\theta}\right] \in H$, we have $\left[X_{-\theta},\left[X_{\theta}, X_{-\theta}\right]\right] \in\left\{X_{-\theta}\right\}$. Q.E.D.

RemarK. If we can prove the Proposition 10 without the hypothesis on the dimension on $L$, then we might be able to classify the infinite primitive Lie algebras.

Now let us go back to our example. The element $X_{3 \alpha+\beta} \in L_{0}$ acts on $L_{(-1)}\left(\bmod L_{0}\right)$ as a matrix of rank 1 . This shows the linear isotropy [5] of $(L, L(\beta))$ is semi-involutive. On the other hand, we have

Proposition 11. Let $\left(L, L_{0}\right)$ be a nonlinear primitive Lie algebra and $M$ be a minimal subspace $\left({ }^{5}\right)$ such that $\left[L_{0}, M\right] \subseteq M, L \supset M \supset L_{0}$. Then the restriction of the linear isotropy of $\left(L, L_{0}\right)$ to $M$ is never semi-involutive.

Proof. If otherwise, the restriction of the linear isotropy to $M$ must be one of $\operatorname{GL}(M), \operatorname{SL}(M), \operatorname{Sp}(M)$ and $\operatorname{CSp}(M)$ (for the notation see [5]) by the classification theorem of the irreducible semi-involutive Lie algebras [5]. But it is easy to see that our linear isotropy is neither of them.

Remark. Lemma 6 in [4] can be easily checked by Proposition 7, Proposition $8\left(8^{\prime}\right)$ and Proposition 6.

APPENDIX. A transitive Lie algebra $\left(L, L_{0}\right)$ is called ample if the normalizer of $L_{p}$ (see $\S 1$ ) in $L$ is identical to $L_{0}$ for any $p \geqq 0$ such that $L_{p} \neq 0$. When $L$ is of infinite dimension, this notion coincides with that of Kuranishi [3]. It is easy to see that any nonlinear primitive Lie algebra is ample. The converse is false. In fact one can easily check that a transitive Lie algebra $\left(L, L_{0}\right)$ such that $L$ is simple and $L_{0}$ is parabolic in $L$, is also ample even if $L_{0}$ is not maximal. We suspect that for any complex ample transitive Lie algebra $\left(L, L_{0}\right)$ such that $L$ is simple, $L_{0}$ is parabolic.

\section{BIBLIOGRAPHY}

1. S. Araki, On root systems and an infinitesimal classification of irreducible symmetric spaces, J. Math. Osaka City Univ. 13 (1962), 1-34.

2. E. Cartan, Les groups de transformations continus, infinis, simples, Oeuvres Complètes. Partie II, Vol. 2, Gau thier-Villars, Paris, 1953, pp. 857-925.

3. M. Kuranishi, The classification of complex simple continuous transitive infinite pseudogroups according to E. Cartan, Mimeographed note, Princeton Univ., Princeton, N. J., 1961.

4. S. Kobayashi and T. Nagano, On filtered Lie algebras and geometric structures. I, J. Math. Mech. 13 (1964), 875-908.

5. —- On filtered Lie algebras and geometric structures. II, III, J. Math. Mech. 14 (1964), 513-521, 679-700.

6. —- On filtered Lie algebras and geometric structures. IV, J. Math. Mech. 15 (1966), 163-171.

7. H. Matsumoto, Quelques remarques sur les groupes de Lie algébriques réels, J. Math. Soc. Japan 16 (1964), 419-446.

(5) I.e. $k=1$ by the meaning of Corollary 9 . 
8. V. V. Morozov, Sur les sous-groupes maximaux non semi-simples des groupes simples, Doctoral Dissertation, Univ. of Kazan, USSR, 1943.

9. C. C. Moore, Compactification of symmetric spaces, Amer. J. Math. 86 (1964), 201-218, 358-378.

10. G. D. Mostow, On maximal subgroups of real Lie groups, Ann. of Math. 74 (1961), 503-517.

11. Séminaire Bourbaki, Sous-algèbre des algèbres de Lie semi-simples, by J. Tits, Exposé $119,1955$.

12. I. M. Singer and S. Sternberg, On infinite groups of Lie and Cartan. I, J. Analyse Math. 15 (1965), 1-114.

UNIVERSITY OF TOKYO,

TOKYO, JAPAN 